\title{
IMPACTO AMBIENTAL Y DESPLAZAMIENTOS FORZADOS EN EL CONTINENTE AFRICANO
}

\author{
Environmental impact and forced displacements \\ in the African continent
}

Giuliana De Battista*

\begin{abstract}
Resumen. Este artículo se propone abordar la temática de los desplazamientos forzados en África, desde un enfoque que, enfatizando en los motivos medioambientales de dichos movimientos, sea capaz de ilustrar la complejidad inherente a este problema, atravesado por una multiplicidad de factores que van desde situaciones políticas, económicas y sociales, a circunstancias étnicas y ecológicas. El objetivo se dirige a cuestionar la todavía no superada distinción entre "migrante" y "refugiado" que subyace al Estatuto sobre los Refugiados de la Convención de Ginebra (1951) y del Protocolo de New York (1967), a partir del señalamiento de los diversos niveles en que los países más desarrollados son responsables de la situación que afecta a estas personas.
\end{abstract}

Palabras claves: migraciones; refugio; ecología; medioambiente; pobreza; conflicto.

\begin{abstract}
This article proposal is to approach the theme of forced displacements in Africa, from a point of view that, emphasizing on the environmental reasons of these movements, may be able to illustrate the complexity of a problem which is crossed by a multiplicity of factors that includes not only political, economical and social reasons, but also etnical and ecological ones. The objective of this paper is to question the not yet overcome distinction between "migrant" and "refugee" that underlies the Statute of Refugees of the Geneva Convention (1951) and the New York Protocol (1967), starting from the indication of the various levels in which the most advanced countries are responsible for the situation that affects these people.
\end{abstract}

Keywords: migrations; refuge; ecology; environment; poverty; conflict.

\footnotetext{
Profesora de Filosofía por la Universidad Nacional de Rosario (UNR). Rosario, Argentina. E-mail:
} gdebattista.unr@gmail.com. Orcid: 0000-0001-9276-9391. 


\section{Introducción}

Los desplazamientos forzados que han tenido, tienen y tendrán lugar en las regiones más desfavorecidas del mundo, constituyen una problemática que exige ser planteada desde diversos puntos de vista, y que condensa muchas cuestiones ligadas al hecho de que hemos creado modos de vida política, económica y medioambientalmente insostenibles. La dimensión que, en la actualidad, adquieren este tipo de migraciones en África -continente con diversos escenarios de conflictos, pobreza estructural e inestabilidad política-, hace de este tópico algo sumamente relevante que ha sido abordado con anterioridad por diversos especialistas ${ }^{1}$.

El objetivo general de este estudio es reflexionar sobre la relación impacto medioambiental-desplazamientos forzados, desde una óptica que busca señalar el vínculo inescindible que los efectos del cambio climático y medioambiental como causantes de la movilidad humana, guardan desde diversas perspectivas con una responsabilidad que compete de manera directa a los países más desarrollados. De manera específica, se tendrá por objeto: 1) analizar la multiplicidad de factores que intervienen y condicionan el vínculo impacto medioambiental-migraciones forzadas; 2) dar cuenta del modo en que, a esta problemática, subyacen una serie de causas más profundas que remiten a aspectos históricos, políticos y económicos del orden internacional vigente; y 3 ) exponer los desafíos que estas situaciones imponen a la comunidad y al régimen jurídico internacional, en particular, al Estatuto sobre los Refugiados de la Convención de Ginebra (1951) y del Protocolo de New York (1967).

\section{Observaciones metodológicas}

Este trabajo se circunscribe al espacio geográfico del continente africano, con especial énfasis en los países del Sahel. Asimismo, se refiere al

1 Ver: Bascom, J., Losing place. Refugee Populations and Rural Transformations in East Africa. Refugee and Forced Migration Studies, v. 3. New York: Berghahn, 1998; Loescher, G., Milner, J., The Long Road Home: Protracted Refugee Situations in Africa. Survival, v. 47, n. 2, p. 153174, 2005; Chatty, D., Finlayson, B. (Eds.) Dispossession and Displacement. Forced Migration in the Middle East and North Africa. Oxford: University Press, 2010; Blanco, P. Migraciones desesperadas en África Subsahariana poscolonial. En: Álvarez Acosta, M. E. (Ed) África Subsahariana: Sistema capitalista y relaciones internacionales. Buenos Aires: CLACSO, 2011, p. 521-541; Couldrey, M. Herson, M. (Eds.) North Africa and displacement 2011-2012. FMR, n. 39, 2012; Okello, J. O. Reflexiones sobre Liberia y Sierra Leona. RMF, n. 52, p. 13, 2016; Kekic, E. Mseke, H. Soluciones de desplazamiento y desarrollo en Tanzania. RMF, n. 52, p. 65, 2016; Majidi, M., Dadu-Brown, S., Soluciones transitorias para los desplazados en el Cuerno de África. RMF, n. 52, p. 66-67, 2016; Patrício, G. V., Peixoto, J. Migração forçada na África Subsaariana: alguns subsídios sobre os refugiados em Moçambique. REMHU, v. 25, n. 54, p. 11-30, 2018; Lopes, C. M. Refugiados, reintegração e mobilidade interna: um olhar sobre o caso angolano 2002-2018. REMHU, v. 25, n. 54, p. 61-76, 2018. 
vínculo que el impacto climático y los desastres naturales establecen con la movilidad humana, refiriéndose el primer término a los cambios que tienen lugar durante un largo período de tiempo en las lluvias y en las temperaturas (promoviendo, por ejemplo, procesos de desertificación), y, el segundo término, a sucesos repentinos e inesperados -como sequías, inundaciones, o huracanes- (Mously Mbaye, 2017). El impacto que poseen los factores climáticos y medioambientales en las migraciones no se concebirá de manera directa (lo cual raras veces acaece en la práctica, presentando estas situaciones diversas complejidades), sino principalmente indirecta, esto es, a través del perjuicio y la alteración que éstos producen en las condiciones de vida de las personas (por ejemplo, a partir de la reducción de la productividad agraria).

Los desplazamientos motivados por factores medioambientales pueden ser tanto internos como internacionales, temporarios como permanentes, dependiendo estas determinaciones -tal como lo demuestran Henry, Schoumaker y Beauchemin (2004)- de diversos factores como la severidad del impacto ambiental en cuestión, las condiciones económicas, y la edad y el género de las víctimas. Existe cierto consenso en torno al hecho de que las migraciones motivadas por factores climáticos ocurren mayoritariamente dentro de las fronteras de los países menos desarrollados, y, en particular, desde las áreas rurales a las urbanas; no obstante, podemos suponer que, con el incremento de las presiones medioambientales, también lo harán los movimientos internacionales (Hugo, 1996). Por este motivo -pero también debido a que, a diferencia de los eventos del tipo rapid-onset, proclives a generar desplazamientos de poca distancia y reasentamientos de escasa duración, los del tipo slow-onset tienden a aumentar la migración externa y permanente (Mously Mbaye, 2017)-, nos interesa poner especial atención en los desplazamientos internacionales vinculados al impacto de factores medioambientales. De allí que se haya establecido como tercer objetivo específico de este escrito el cuestionamiento de la adecuación de la Convención de Ginebra y del Protocolo de New York a diversos escenarios de la realidad actual: quienes cruzan la frontera debido al modo en que los factores medioambientales impactan en sus condiciones de vida, se exponen a un limbo legal que vulnera ampliamente los derechos humanos de las personas.

El presente estudio constituye una investigación cualitativa, y descriptivaexplicativa. Se utilizará una técnica bibliográfica-documental, así como procedimientos comparativos y tipológicos, para avanzar en la consecución de los objetivos establecidos a partir de un enfoque dialéctico. 


\section{Naturaleza de la relación entre el impacto medioambiental y la movilidad humana}

El título del presente artículo no pretende sugerir un esquema de análisis monocausal en virtud del cual sería posible distinguir migraciones motivadas única y exclusivamente por factores medioambientales, sino dilucidar la importancia que revisten estos últimos en la medida en que son "parte de complejos patrones de causalidad múltiple, en los cuales los factores naturales y medioambientales están estrechamente vinculados a otros factores económicos, sociales y políticos" (Castles, 2002, p. 5)².

La relación instrumentalista que el ser humano ha establecido con la naturaleza desde hace aproximadamente doscientos años, ha conducido a una situación que se nos revela claramente insostenible y que impacta en la vida de millones de individuos. Tal cosa contribuye en gran medida (toda vez que estos factores se combinan de determinada manera con otros de índole política, social y hasta personal) a que tengan ocasión diferentes tipos de desplazamientos, con respecto a los cuales tenemos buenas razones para considerar que se diferencian de algo que, a lo largo de la historia, ha constituido una mera estrategia de supervivencia:

La respuesta de abandonar un área de forma permanente o temporal ante una crisis ambiental que pone en peligro la vida es, por supuesto, eminentemente racional y ha sido la estrategia de supervivencia más importante a lo largo de la historia humana. Sin embargo, la situación a mediados de la década de 1990 con respecto a tales movimientos parecería diferir en cierta medida de la experiencia histórica en varias formas: la escala y el ritmo del cambio ambiental ha aumentado sustancialmente; las diferencias entre los países en la capacidad de hacer frente a estos cambios ha aumentado. (Hugo, 1996, p. 113) ${ }^{3}$

Entre el entorno selvático de los países del Golfo de Guinea y el entorno desértico de los países del Sahel, avanza el Desierto del Sahara. En el Lago Chad, por ejemplo, la cantidad de agua se ha reducido gravemente desde las últimas cinco décadas debido a la disminución de las lluvias y a una mayor evaporación de las mismas producto del calentamiento global: "Ese entorno del Lago Chad, aparte de su exigua agricultura, disponía de pastos y malezas que servían de alimento a poblaciones humanas y al ganado. En la actualidad, en toda esa cuenca (...) unos diez millones de personas se encuentran en situación de hambruna" (CEAR, 2018, p. 15). No obstante, tal como nos lo advierten McLeman y Smit (2006), no siempre es fácil distinguir si este problema es consecuencia de la inequidad económica y política que afecta el acceso al alimento, o la consecuencia de la alteración en las lluvias y de los cambios medioambientales.

\footnotetext{
${ }^{2}$ La traducción del inglés al castellano de todas las citas de este texto es propia.

${ }^{3}$ La traducción del inglés al castellano de todas las citas de este texto es propia.
} 
Hugo (1996), refiriéndose a la zona del Sahel, afirma que las sequías que tuvieron lugar en el período que va de 1968 a 1973 y de 1982 a 1984 derivaron en millones de desplazamientos forzados allí cuando, únicamente en el primero de estos dos períodos, hubo 1 millón de "refugiados medioambientales" solo en Burkina Faso. Hacia finales del segundo período se registraron las siguientes cifras: 222.000 desplazados en este último país; 500.000 en Chad; 200.000 en Mali; 190.000 en Mauritania; y 1.000.000 en Níger. Henry, Schoumaker y Beauchemin (2004) realizan un estudio en torno al impacto de los cambios de las lluvias en los suelos de Burkina Faso, uno de los países más pobres del mundo, con una bajísima tasa de urbanización, y en el que la economía se encuentra ampliamente basada en la actividad agrícola y ganadera (constituyendo el $90 \%$ de las actividades desarrolladas por su población). Tratándose, en gran medida, de una agricultura de subsistencia, las condiciones agro-climáticas que afectan su productividad -principalmente en el norte del país- se traducen en diversos tipos de migraciones: a países vecinos como Costa de Marfil y Ghana, o al interior del país, ya sea desde las áreas rurales a las urbanas, o, en mayor medida, hacia otras áreas rurales (Henry et alii, 2004).

La erosión de los suelos y la desertificación -además de producirse por el cambio climático, la deforestación, y la implementación de determinadas formas de agricultura y ganadería- también se origina por los cultivos extensivos diseñados para conseguir altas producciones de un único recurso, agrícola o ganadero; suelen ser industrias de exportación, y requieren grandes cantidades de agua y de abonos químicos y fitosanitarios (CEAR, 2018). En el sur de Costa de Marfil la llamada "ruta del cacao" se vio desplazada desde el este hacia el oeste del país a causa del agotamiento del suelo, y de la consecuente presión de los flujos migratorios internos: "en la extensión de las plantaciones de cacao, café y algodón, habidas en las pasadas décadas, en Costa de Marfil se ha perdido el 80\% de la selva original" (CEAR, 2018, p. 49). Los procesos de deforestación que han tenido lugar en este país a lo largo de los años para dar lugar a dichos macrocultivos fueron denunciados por la ONG Mighty Earth que, en su Informe Déforestation amère du chocolat (2016), afirma que miles de chimpancés, elefantes y otras especies fueron diezmadas a partir de la conversión de bosques en plantaciones de cacao. También la organización Farmlandgrab (2017) ha denunciado los severos problemas que se siguen de la sobreexplotación de los suelos a cargo de empresas transnacionales como SOCFIN Group en Nigeria, Camerún, Sierra Leona y Liberia; dichos problemas incluyen, no sólo daños medioambientales, sino también la expropiación de tierras, la explotación laboral, y hasta la persecución y encarcelamiento de líderes comunitarios a causa del cultivo de palma de aceite y árboles de caucho. 
Ahora bien, ¿cuál es el tipo de relación que estos sucesos guardan con las migraciones de las personas si, tal como ya lo establecimos, éstas no pueden ser consideradas como una respuesta automática a los riesgos que se siguen de los cambios climáticos y medioambientales? McLeman y Smit (2006) proponen que la relación clima-migración se inserta en un modelo más general construido en torno al concepto de vulnerabilidad, allí donde esta última es inteligible a partir del elemento de la exposición, y de la capacidad de adaptación, siendo $V=(E, A C)$. En este escenario, la migración constituye una potencial vía de adaptación a los cambios o eventos climáticos que depende del nivel de exposición frente a dichos eventos, y de las capacidades efectivamente existentes de adaptación, allí cuando éstas pueden ser analizadas tanto a nivel individual como a nivel institucional (McLeman, Smit, 2006). Esto último significa que la reacción o la respuesta de la gente ante estos sucesos pocas veces asume el control efectivo sobre la situación, y que la decisión o la imposición que deriva en el desplazamiento o en la permanencia, depende de determinados bienes disponibles, pero también de procesos sociales y políticos más amplios: "las características particulares de la capacidad de adaptación diferencia a los migrantes de los no-migrantes" (McLeman, Smit, 2006, p. 46) ${ }^{4}$. En este sentido, y dependiendo de lo que estos autores denominan capital endowments, es necesario tener en cuenta que no todos los potenciales migrantes deciden desplazarse (si consideran, por ejemplo, que existen otras alternativas de adaptación preferibles), pero también que no siempre existe la opción de la permanencia ("algunos hogares pueden no tener otra opción más que migrar" [McLeman, Smit, 2006, p. 46]), o, al contrario, no siempre la migración es una opción disponible. Este último punto es abordado por Black (2011) cuando, refiriéndose al modo en que el cambio medioambiental alterará en el futuro los patrones migratorios, también busca inteligir la migración a partir de su carácter adaptativo, tendiendo a desmitificar su visión apocalíptica y negativa. Los mayores riesgos recaerían sobre aquellos incapaces de relocalizarse, y serían exacerbados por las políticas destinadas principalmente a prevenir la migración (Black et alii, 2011). Desde esta óptica, es necesario considerar que el impacto de los cambios medioambientales no sólo puede incentivar la movilidad humana, sino también limitarla o imposibilitarla:

\footnotetext{
No todos pueden migrar. Pueden existir factores sociopolíticos confusos, como en Somalia, donde los conflictos armados restringen el movimiento (...). La migración a menudo es costosa, y los más vulnerables al cambio ambiental suelen ser pobres (...) en Uganda (...) los que son más ricos son capaces de reubicarse. En Malí, la emigración disminuyó durante las severas sequías de 1983-85, junto con un aumento de la pobreza rural. (Black et alii, 2011, p. 448) ${ }^{5}$
}

\footnotetext{
${ }^{4}$ La traducción del inglés al castellano de todas las citas de este texto es propia.

${ }^{5}$ La traducción del inglés al castellano de todas las citas de este texto es propia.
} 
El hecho de que los procesos ambientales muchas veces pueden retraer, en lugar de acentuar, los flujos migratorios, también se explica por el carácter mismo de los eventos de inicio lento: "cuando se hace frente a eventos del tipo rapid-onset, tales como ciclones, tormentas, e inundaciones, las personas pueden verse forzadas a moverse porque pierden todo de repente, lo cual no es necesariamente el caso en los eventos del tipo slow-onset" (Mously Mbaye, 2017 , p. 6$)^{6}$.

\subsection{Violencia, conflicto, e impacto medioambiental}

Según la Escola de Cultura de Pau (ECP, 2018) en el continente africano tiene lugar la mayor cantidad de conflictos activos en el mundo. Estos últimos reflejan escenarios con denominadores comunes y elementos diferenciales, en los que se observan situaciones de inestabilidad política, conflictos étnicos -la población albina se halla en constante peligro en países como Malawi o Tanzania, por ejemplo-, religiosos, y de género. Asimismo, tiene lugar la presencia de grupos militarizados -grupos yihadistas activos en Mali (Estudios de Política Exterior, 2017), Boko Haram en Nigeria, Al Shabaab en Somalia, etc.- que ejercen todo tipo de violencias contra la sociedad civil y personal de ayuda humanitaria, buscando el control territorial y la explotación ilegal de recursos (CEAR, 2018).

En escenarios que promueven los desplazamientos de las personas, las situaciones de violencia y conflicto muchas veces se hallan complejamente imbricadas con las problemáticas medioambientales. Castles (2002) se propone desbaratar la hipótesis según la cual estas últimas constituyen la causa de conflictos que, a su vez, derivan en desplazamientos internos y en masas de refugiados. Así, afirma que otros factores, "tales como las divisiones políticas, las rivalidades étnicas y los intereses económicos parecen mucho más importantes como causantes de la violencia y la guerra" (Castles, 2002, p. 7). Black (1998) afirma que gran parte de los conflictos de la década de los 90 estaban promovidos por el control sobre valiosos recursos como el petróleo, y no debido a la escasez de estos últimos. Desde la óptica adoptada en el presente escrito, consideramos que existen buenas razones para pensar que es precisamente este último punto, a saber, el afán de control sobre los recursos, el que se verá exasperado ante el inminente aumento de las presiones medioambientales ("que las guerras siempre han tenido que ver con asuntos similares [recursos de todo tipo] es obvio, pero que las alteraciones bio-geo-físicas y climáticas contemporáneas aumentan el riesgo y exasperan los conflictos es algo no menos claro" [Espinosa, 2011, p. 118]).

${ }^{6}$ En 2016 la ciudad de Lagos en Nigeria se debatía entre "defenderse de los ataques del océano y las lluvias torrenciales o concebir el agua como una parte integral de la ciudad" (El País, 2016). En contextos de este tipo han de concebirse los denominados rapid-onset events. 
Aun asumiendo la complejidad de determinados escenarios en los que ni siquiera es fácilmente distinguible cuál es el verdadero desencadenante del conflicto (como en Ruanda 1994, desastre al que subyacen motivos étnicos, medioambientales y fundamentalmente políticos), es importante retomar un planteo como el de Welzer (2010). El autor afirma que Darfur, al oeste de Sudán, es un claro ejemplo del modo en que el cambio climático puede constituir un aspecto clave en la emergencia de conflictos, refiriéndose a los sucesos acaecidos en dicho espacio geográfico como a "la primera guerra climática" (aun cuando a los occidentales nos haya sido presentado como un enfrentamiento meramente étnico entre pastores árabes y agricultores africanos). Este escenario incluye diversos elementos y circunstancias que son susceptibles de ser explicados a través de las nociones con las que Welzer intenta inteligibilizar estos contextos, esto es, los conceptos de "guerra climática", "guerra permanente", "mercado de la violencia", y "estado fallido": en muchos conflictos y guerras actuales las partes involucradas no tienen ningún interés en que éstas terminen debido a que existen importantes intereses políticos y financieros que responden a un verdadero "mercado de la violencia", allí cuando las débiles estructuras políticas y económicas de determinados países, lejos de emprender acciones para monopolizar la violencia y para encarnar el estado de derecho, contribuyen a empeorar y perpetuar esta situación, dejando un espacio libre para el desarrollo de estos circuitos y actividades ilícitas (Welzer, 2010).

Dilucidar la lógica que atañe a estos conflictos no es un designio sencillo y no admite explicaciones simples, siendo la mayoría de las veces el producto de diversos procesos paralelos. No obstante, sí es posible afirmar que "las causas estructurales del conflicto, como la desestatización, el surgimiento de mercados de violencia y la exclusión o el exterminio de algunos grupos de la población, se acentúan y aceleran con los problemas ecológicos y con la desaparición de recursos" (Welzer, 2010, p. 127).

\section{Una mirada hacia atrás, y hacia el presente: las presiones medioambientales desde la óptica de los neocolonialismos}

Las fronteras de África no fueron trazadas geográficamente, sino 'geométricamente', en un procedimiento que revistió el carácter de un verdadero 'reparto'(Arancón, 2015). En el siglo XIX muchos países europeos se lanzaron hacia la conquista de estos territorios, teniendo todas las metrópolis preferencias por aquellos que tuviesen fácil acceso, potencial explotación económica, y mercados propicios para introducir las manufacturas europeas. Fue por eso necesario organizar una conferencia dirigida a organizar la expansión colonial, esto es, a 'repartir el pastel', estableciendo los límites que le correspondían a cada potencia, en un arbitrario ejercicio fronterizo 
en virtud del cual todavía se explican muchos de los conflictos que ha sufrido el continente desde la segunda mitad del siglo XX (Arancón, 2015). Tal fue la tarea que tuvo la Conferencia de Berlín, convocada en 1884 por Bismarck. Como es posible imaginar, este procedimiento constituyó una decisión puramente política que no tuvo en cuenta la geografía física, ni el hecho de que se estaban dividiendo territorios y poblaciones más o menos homogéneas -como es el caso del Marruecos hispano-francés o la Somalia partida entre franceses, británicos e italianos-, al mismo tiempo en que se agrupaban bajo un mismo territorio grupos étnicos cuya relación no era precisamente cordial.

Tampoco debemos olvidar que el estado de gran vulnerabilidad en que deja a los países africanos el proceso de descolonización, se transformó rápidamente en la contracción de una deuda externa en constante aumento gracias a la adquisición de créditos concedidos por los países del Norte en la forma de políticas de Ayuda al Desarrollo: "Para los países de África del Norte, del África subsahariana y de Oriente Medio, los montos totales de la deuda externa se multiplicaron por 73 entre 1970 y 2012. Durante ese período, estos países pagaron 145 veces la cantidad inicial" (CADMT, 2015). Este escenario refleja clara y desafortunadamente aquello a lo que J. Ziegler se refiere cuando afirma que "el medio de dominación más poderoso es actualmente el servicio de la deuda" (El Salto, 2018). En 2016 un comité consultivo del que este último es el vicepresidente, presentó ante Naciones Unidas un informe sobre los llamados holdouts o fondos buitre. Además del caso argentino, incluye el de dos países del continente africano, Zambia y República Democrática del Congo, dando cuenta del modo en que las actividades de los fondos buitres disminuyen la capacidad de los Estados para garantizar los derechos económicos, sociales y culturales, ponen en peligro las iniciativas internacionales de reducción de la pobreza, contribuyen a aumentar el servicio de la deuda, y menoscaban la consecución de los objetivos de desarrollo sostenible (Asamblea General De Naciones Unidas, A/HRC/33/54).

El abordaje medioambiental del asunto de las migraciones forzadas no ha de concebirse independientemente de las dinámicas neocoloniales que rigen actualmente el orden internacional. El cambio climático es, no sólo un elemento que siempre ha sido parte del desarrollo humano, sino principalmente la consecuencia de la falta de políticas verdaderamente vinculantes que comprometan a los Estados a regular y limitar su impacto en el medioambiente (a partir, por ejemplo, de la disminución de la emisión de gases de efecto invernadero), pero también la consecuencia de un modelo económico extractivo basado en la sobreexplotación de recursos naturales, y en la proliferación de megaproyectos de empresas trasnacionales. El énfasis puesto en la relación migración forzada-crisis ecológica, más que constituir una distracción de los problemas centrales del desarrollo y la resolución de 
conflictos (tal como ha sido percibido por algunos especialistas), se encuentra estrechamente relacionado con estos últimos. Es lo que, en cierto sentido, Castles afirma cuando alude a la necesidad de resituar el vínculo impacto ambiental-migración forzada en el marco de las relaciones de poder globales y de la división norte-sur:

(...) los países del norte son responsables de la mayor parte de los problemas medioambientales. Esto aplica en un sentido evidente: el estadounidense promedio produce diez veces más gases de efecto invernadero y calentamiento global que el hindú o chino promedio. Pero también es cierto de una manera mucho más penetrante. La globalización como nuevo orden mundial se basa en la apertura de todas las regiones del mundo a actividades económicas en gran parte controladas por las empresas transnacionales con sede en el Norte y motivadas por sus intereses de lucro. Las instituciones económicas globales -el FMI, el Banco Mundial y la Organización Mundial de Comercio-, imponen condiciones de privatización, libre comercio, e inversión y control sobre la propiedad intelectual que protege el dominio de las transnacionales. (Castles, 2002, p. 11)

El problema de muchos de los países del continente africano es la dificultad para aprovechar el potencial de sus recursos a través de tecnologías sustentables, y crear ciclos de transformación interna hacia productos finales con mayor valor añadido que los desplacen de su papel de exportadores de materias primas ${ }^{7}$. Es necesario establecer una lucha contra los flujos comerciales que, a través del mantenimiento de una lógica neocolonial, perjudican a la ciudadanía de estos países, e impedir que la relación de las potencias con estas regiones sea la de una mera fuente de abastecimientos que no repara en el impacto medioambiental que se sigue de la explotación indiscriminada de los recursos. Según Casa África/España (2017), una de las principales conclusiones a las que se arriba en Perspectivas Económicas en África 2017, es la de que hay que liberar el potencial de los empresarios africanos para acelerar la transformación industrial del continente.

No obstante, el reconocimiento de la necesidad de avanzar en esta dirección, no debe conducirnos a ignorar que este asunto no descansa únicamente en una cuestión de discernimiento político, sino también en el escaso margen de libertad que poseen los gobiernos para implementar medidas que muy rápidamente son denunciadas como 'proteccionistas' ante los tribunales de la Organización Mundial del Comercio (OMC). Este punto es

\footnotetext{
Ya hemos hecho referencia a la estructura económica principalmente agrícola y ganadera de Burkina Faso. La CEAR (2018) aporta los siguientes datos: Camerún, país significativo en la exportación granos de cacao, no tiene fabricación propia de chocolate, por lo que en sus puestos comerciales se encuentran chocolates suizos, belgas y británicos; Costa de Marfil tiene ingresos de 4 mil millones al año por la exportación de granos y manteca de cacao, cifra muy inferior a la de cada una de las grandes empresas de chocolate europeas o estadounidenses; Mali es uno de los exportadores más importantes de algodón en la región subsahariana, pero son los organismos comerciales los que regulan el precio, por lo que los campesinos y campesinas usualmente se ven obligados a abaratar los precios para poder venderlos.
} 
abordado extensamente por Klein en su obra Esto lo cambia todo (2015), en la que hace alusión a la multiplicidad de denuncias mutuas que países y alianzas internacionales han Ilevado ante aquella organización, cada vez que se ha intentado apostar al desarrollo de energías renovables a través de proyectos que suponen una promoción de ciertas industrias locales. Desde el punto de vista climático los dictámenes de la OMC son algo absurdo y disparatado; pero los argumentos presentados en aquellas ocasiones eran totalmente coherentes desde la perspectiva jurídica-legal (Klein, 2015). En este escenario, nos vemos acorralados por los pactos comerciales y por el marco normativo de un derecho mercantil -cuyo principal motor fue responder a los intereses de grandes multinacionales- que vuelve estériles en la práctica muchas de las negociaciones relativas a un desarrollo sostenible (Klein, 2015).

Tal como se observa, la posibilidad de que los países africanos implementen políticas dirigidas a lograr un desarrollo interno sostenible (único proceso que contribuiría a aminorar los graves problemas medioambientales que sufre la mayor parte de éstos, a la vez que a propiciar cambios en su estructura económica), se ve limitada de antemano por un contexto internacional neoliberal que, en nombre del libre comercio, prescribe la desregulación del sector privado empresarial que obstaculiza las apuestas por las energías renovables y perpetúa un modelo por el cual las periferias subdesarrolladas continúan respondiendo a los centros industrializados. Pero, siguiendo esta perspectiva largoplacista, nuestros esfuerzos también se han de dirigir a la sustitución de la racionalidad científica-técnica-instrumental, por una razón ecológica capaz de sustentar una transformación estructural en nuestro estilo de vida (Espinosa, 2011). Desde una perspectiva ético-jurídica, esta última se ha de materializar en un modelo de ciudadanía que establezca otro tipo de relación con el medio en el cual vive. Las teorizaciones de Dobson (2005) en torno a la idea de una ciudadanía ecológica y desterritorializada -susceptible de volverse inteligible a partir del concepto de huella ecológica-, pueden ser de suma utilidad para avanzar en este camino.

\section{Refugiados vs. Migrantes ambientales}

En la extensa literatura existente sobre migraciones abundan las tipologías que diferencian a los migrantes y a las migraciones a partir de diversos criterios. Muchos autores identifican a la figura del refugiado a partir de modelos que se ciñen al Protocolo de New York (1967) y a la Convención de Ginebra (1951) -limitando dicho concepto al reconocimiento de las presiones políticas y de los conflictos como causantes del desplazamiento-, o a partir de modelos más inclusivos que, entre dichos motivantes, incluyen la insuficiencia económica ${ }^{8}$.

${ }_{8}$ Ver: Olson, M. E., "Refugees as a special case of Population Redistribution". En Population 
Castles (2002) -que sostiene que el término refugiado medioambiental es simplista, engañoso y confuso, puesto que implica una visión monocausal que rara vez existe en la práctica- afirma que, dado el actual clima de hostilidad hacia el solicitante de asilo, y la falta general de consenso para ampliar el estatuto del refugiado, cualquier cambio que se realice sobre este último sea probablemente para peor. De este modo, afirma que "necesitamos hacer nuestro mayor esfuerzo para proteger la Convención de 1951, reclamando, al mismo tiempo, por mejores instituciones y regímenes legales internacionales para proteger a otros tipos de migrantes forzados" (Castles, 2002, p. 10) . La $^{2}$ OIM (2011) habla de migrantes ambientales, definiéndolos como "personas o grupos de personas que, por razones imperiosas de cambios repentinos o progresivos en el medioambiente, que afecten negativamente su vida o sus condiciones, se ven obligados a abandonar su lugar de residencia habitual o deciden hacerlo, ya sea temporal o permanentemente, ya sea en su país o en el extranjero". La CEAR, por su parte, propone que dentro de la categoría de desplazamiento forzado por causas medioambientales "existen casos a los que sí se podría aplicar el marco jurídico de protección, por ejemplo, en los movimientos de personas a causa de conflictos armados enraizados en factores ambientales" (CEAR, 2018, p. 77).

A nuestro juicio, estas formulaciones -que equivalen a afirmar que el status de refugiado ha de contemplar a las personas que huyen de la guerra o de la violencia pero no a las personas que huyen de la pobreza y de peligros que amenazan su integridad física- simplemente no alcanzan. No hacen justicia suficiente a la conexión existente entre pobreza y herencias coloniales o neocolonialismos propios de un modelo económico neoliberal, y a la conexión existente entre pobreza y crisis ecológica, allí cuando las consecuencias de esta última (que, por supuesto, no se limitan a la pobreza) son estremecedoramente más graves en los países que carecen de los recursos necesarios para adaptarse a los efectos de algo a lo que apenas contribuyen ${ }^{10}$. No obstante, son los que soportan las mayores cargas de los daños que producen otros: otros que tienen mejores niveles de vida y capacidades de consumo, otros que no toman las medidas necesarias, otros que esconden y niegan la urgencia del problema,

Redistribution: Patterns, Policies and Prospects. Ed. L. A. P Gosling and L. Y. C. Lim. New York, 1979, p. 130-152.

9 Existen diversos tipos de protección internacional entre los cuales el status de "refugiado" es el que, por así decir, otorga más derechos y posibilidades de inserción local a las personas.

${ }^{10}$ Dado el poco peso que posee la industria en la estructura económica de los países africanos, éstos son los que menos emisiones de GEl producen. En Nigeria el esquema de Consumo Final de Energía (CFE) guarda una relación desproporcionada con la producción de petróleo y derivados que existe en el país. Sólo se consume el $10 \%$ de lo que se produce, el resto es de íntegra exportación, lo cual se refleja en el hecho de que sólo tiene acceso a la electricidad entre un $40 \%$ y un $60 \%$ de la población (CEAR, 2018). 
y otros que los producen tanto en sus países industrializados como en las tierras africanas explotadas. Quienes más se preocupan por su 'seguridad nacional' -la resolución 427 de la OTAN, insta a los gobiernos a reconocer que los riesgos asociados al cambio climático son importantes multiplicadores de las amenazas, en tanto que exacerban la presión migratoria (Cubel, 2017)no están dispuestos a reconocer los diferentes y múltiples niveles en que son partícipes de un asunto que afecta directamente a los derechos humanos de las personas. Esta falta de aceptación de la responsabilidad que les compete a muchos y esta falta de compromiso político con la humanidad es la que se refleja en las siguientes cifras: en 2014 el $86 \%$ de los refugiados eran acogidos en países en vías de desarrollo (es sabido que, en África, la gran mayoría busca refugio en países fronterizos), y de las 570.000 peticiones de asilo registradas en la UE, sólo 161.000 recibieron algún tipo de protección internacional (EL PAÍS, 2015).

Desde la óptica del presente estudio, consideramos que es necesario avanzar en el perfeccionamiento del derecho internacional, y no adecuarnos a restricciones jurídicas que no reconocen las complejas características de determinadas realidades espacio-temporales. No sólo porque la mayoría de las personas que necesitan protección internacional no son admitidas en la categoría de refugiados, sino porque esta última, tal como es formulada en la Convención de Ginebra, no contempla la dificultad para determinar la naturaleza propiamente política de los motivos del exilio que justifican el pedido de asilo, e ignora hasta qué punto la insatisfacción de las necesidades básicas constituye un motivo de huida forzosa: "La definición de refugiado esconde un 'prejuicio liberal' según el cual es posible, deseable y legítimo diferenciar los problemas políticos, religiosos, étnicos, culturales y sexuales, de los problemas económicos y sociales" (Penchaszadeh, 2010, p. 64). Las violaciones graves a los Derechos Económicos Sociales Culturales y Ambientales (DESCA) han de concebirse como un tipo de persecución, y nuestra tarea ha de ser la de avanzar hacia formulaciones más inclusivas del régimen de protección internacional, y del Estatuto sobre los Refugiados en particular, tal como, por ejemplo, se promueve desde la Iniciativa Nansen (Cubel, 2012). En este sentido, la noción de refugiado medioambiental, aun haciendo énfasis en el motivo medioambiental del desplazamiento, no implica (al menos no necesariamente) un abordaje monocausal del asunto, sino que expone el carácter restrictivo del derecho al refugio tal como éste permanece formulado en la actualidad (aun cuando, desde un análisis radical, sea posible afirmar que -como disposición jurídica creada en 1951- el refugio en sí mismo pervierte el espíritu de la práctica pre-estatal e 'incondicional' del asilo, anteponiendo el derecho soberano de recibir al derecho a ser recibido [Penchaszadeh, 2010]). 
Por supuesto, la creación de una nueva (o extendida) categoría jurídica y, con ella, la de un marco de protección superador de las actuales políticas migratorias, no es un designio sencillo. En primer lugar, porque desafía al andamiaje teórico vigente en tanto que "el art. 10 de la Convención de 1951, no cuenta con resquicios legales que permitan 'elastizar' o flexibilizar sus términos para incorporar a los 'refugiados climáticos'” (Odriozola, 2017, p. 100); y, en segundo lugar, porque requiere el acuerdo sobre una definición, y el establecimiento de los límites y elementos que la componen, en un contexto que se encuentra muy lejos de alcanzar un consenso en torno a este asunto. No obstante, este es el camino que debemos seguir si queremos hacer frente al 'limbo legal' en el que se encuentran aquellos que, motivados por las presiones medioambientales, se ven forzados a cruzar una frontera.

\section{A modo de conclusión}

Tal como ha sido analizado hasta aquí, los cambios climáticos y medioambientales efectivamente impactan en las condiciones de vida de las personas alterando los patrones de la movilidad humana, aun cuando no sea posible adoptar la lógica de una causalidad simple y única para avanzar en la comprensión de estos sucesos. De allí que haya sido necesario especificar ciertos casos en los que las migraciones -en tanto que respuesta adaptativa y privada, cuando las instituciones de la comunidad no son capaces de producir por sí mismas las condiciones de dicha adaptación- no son posibles, pudiendo el impacto de los factores medioambientales retraer la movilidad de las personas. Como hemos visto, estas situaciones pueden tener lugar a partir del desencadenamiento de eventos del tipo slow-onset, de la existencia contextos de violencia que impiden el movimiento, o de la falta de medios económicos o de redes familiares por parte de los/as afectados/as. Asimismo, permaneciendo en el marco de la comprensión del nexo impacto medioambiental-migración forzada, hemos afirmado que los problemas ecológicos y la escasez de recursos exacerban la emergencia de conflictos, aun cuando las evidencias nos previenen de adoptar una visión reduccionista que presuponga que el impacto medioambiental conduce rápidamente a conflictos que derivan de manera directa en migraciones forzadas.

Por otro lado, el re-posicionamiento del abordaje medioambiental de las migraciones forzadas al interior de las relaciones de poder que, en el ámbito internacional, perpetúan los colonialismos, ha contribuido al señalamiento de las causas profundas que subyacen a esta problemática. De este modo, se ha expuesto la magnitud de la responsabilidad que, desde diferentes perspectivas, compete a los países más desarrollados: no sólo porque las presiones medioambientales que afectan las condiciones de vida de las personas recaen principalmente sobre los países que no las producen en un 
nivel significativo (dado el escaso nivel de consumo de su ciudadanía, y dada la estructura principalmente primaria de su economía), sino también porque la incapacidad de estos países para hacer frente a dicho impacto tiene su causa en un modelo económico neoliberal que menoscaba la posibilidad de su desarrollo sostenible, ya sea a través del funcionamiento de determinadas dinámicas comerciales, o a través de mecanismos de dominación como el de la deuda. Eso por no mencionar el hecho de que, en gran medida, es la actividad de empresas transnacionales con sede en el norte la que contribuye a la erosión de los suelos y al avance de la desertificación, además de explotar la mano obra de estos países.

El énfasis puesto en la dimensión de la responsabilidad ha servido como telón de fondo para exacerbar el carácter ya de por sí restrictivo de la definición del refugio vigente en la normativa internacional: además de no reconocer hasta qué punto la insatisfacción de las necesidades básicas (difícilmente distinguible de los motivos sí reconocidos por dicha normativa) puede constituir una causa de persecución, ignora que, a los problemas medioambientales originados en el contexto internacional, corresponde una respuesta de la comunidad internacional. La ampliación del Estatuto sobre los Refugiados es una demanda de carácter urgente, y una de las tantas acciones que, en el corto plazo, contribuiría a la protección de los derechos de las personas que, debido al modo en que las presiones medioambientales afectan sus condiciones de vida, cruzan una frontera.

La migración, además de constituir un derecho (aspecto hoy en día tan frecuentemente olvidado), ha de concebirse como una vía de adaptación inmediata a los cambios medioambientales. Como tal, la prioridad debería ser la habilitación de canales migratorios que le reconozcan su carácter forzado, y no la prevención de las mismas.

\section{Bibliografía}

\section{Información periodística e institucional}

'Apartheid' climático. El País. Lagos (Nigeria). 19.02.2016. Disponible en: < https:// elpais.com/elpais/2016/01/11/planeta_futuro/1452536004_523770.html>. Acceso en: 15.02.2019.

ARANCÓN, Fernando. Los caprichos fronterizos de África. El Orden Mundial (EOM). 2015. Disponible en: <https://elordenmundial.com/los-caprichosfronterizos-de-africa/>. Acceso en: 02.03.2019.

CADMT (Comité para la Anulación de las Deudas Ilegítimas). Las cifras de la deuda 2015. Cap. 3. La deuda en el Sur. Disponible en:

$<$ http://www.cadtm.org/spip.php?page=imprimer\&id_article=12976>. Acceso en: 20.01.2019 
CASA ÁfRICA. Perspectivas Económicas en África. 2018. Disponible en: $<$ http://www.casafrica.es/perspectivas_economicas_africa.jsp >. Acceso en: 20.01.2019.

CEAR (Comisión Española de Ayuda al Refugiado). Refugio por causas ambientales: África en el olvido. Estudio de Casos. 2018. Disponible en: <https://www. cear.es/wp-content/uploads/2018/10/Africa-en-el-olvido.pdf $>$. Acceso en: 15.12.2018.

Convención de Ginebra sobre el Estatuto de los Refugiados. 1951. Serie Tratados de Naciones Unidas, v. 189, n. 2545.

CUBEL, Fernando Martín. El esfuerzo por el reconocimiento del refugiado ambiental: la Iniciativa Nansen. Instituto Español de Estudios Estratégicos (ieee.es). 2017. Disponible en: <http://www.ieee.es/Galerias/fichero/docs_opinion/2017/ DIEEEO03-2017_IniciativaNansen_MartinCubel.pdf >. Acceso en: 07.03.2019. ECP (Escola Cultura de Pau). Alerta 2018! Informe sobre conflictos, derechos humanos y construcción de paz. 2018. Disponible en: <https://escolapau. uab.cat/img/programas/alerta/alerta/18/TodasInfografias.pdf $>$. Acceso en: 20.2.2018.

FARMLANDGRAB. SOCFIN's plantations in Africa: Many places of violence and destruction. 2017. Disponible en: <https://www.farmlandgrab.org/post/ view/27491>. Acceso en: 17.01.2019.

Fondos Buitre. La plutocracia contra el pueblo. El Salto, 01.01.2018. Disponible en: < https://www.elsaltodiario.com/fondos-buitre/naciones-unidasplutocracia-contra-pueblo?fbclid = IwAR0kbvCQU1VHt-EoYthvIIITUXPwXAJEeer_48tKOogIXnvg0SIAH4btx8>. Acceso en: 28.02.2019.

Informe del Comité Asesor del Consejo de Derechos Humanos sobre las actividades de los fondos buitre y sus repercusiones en los derechos humanos. 2016. Asamblea General de las Naciones Unidas, A/HRC/33/54.

MIGHTY EARTH. La déforestation amère du chocolat. 2016. Disponible en: <http://www.mightyearth.org/wp-content/uploads/2016/07/chocolates_dark_ secret_francais_web.pdf $>$. Acceso en: 18.01.2019.

OIM (Organización Internacional de las Migraciones). Portal sobre la migración por motivos ambientales. 2011. Disponible en: < https://environmentalmigration. iom.int/es/migraci\%C3\%B3n-por-motivos-ambientales $>$. Acceso en: 01.03.2019.

Protocolo de New York sobre el Estatuto de los Refugiados. Serie Tratados de Naciones Unidas № 8791, v. 606. 1967.

Un mundo de refugiados. El País, 06.02.2017. Disponible en: < https://elpais.com/ especiales/2015/refugiados/?fbclid=IwAR14RErciKhZSCKxWBQ--ymZ4Y9Znh quE645iNUcfMFu1 pcb5eVu8myiuwA>. Acceso en: 26.02.2019.

Zonas calientes 2017: África (II). Estudios de Política Exterior, 05.04.2017. Disponible en: <https://www.politicaexterior.com/actualidad/zonas-calientes2017-africa-ii/>. Acceso en: 02.03.2019. 


\section{Bibliografía académica}

BLACK, Richard. Refugees, Environment and Development. London: Longman, 1998.

BLACK, Richard et alii. Climate change: migration as adaptation. Nature, v. 478, n. 7370, p. 477-479, 2011.

CASTLES, Stephen. Environmental change and forced migration: making sense of the debate. Working Paper No. 70. Geneva: UNHCR, 2002.

DOBSON, Andrew. Ciudadanía ecológica. ISEGORÍA, n. 32, p. 47-62, 2005.

ESPINOSA RUBIO, Luciano. Naturaleza e historia hoy: la crisis ecológica. Azafea, Rev. filos., n. 13, p. 109-129, 2011.

HENRY, Sabine et. alii. The impact of rainfall on the first out-migration: A multilevel event-history analysis in Burkina Faso. Population and Environment, v. 25, n. 5, p. 423-460, 2004.

HUGO, Graeme. Environmental concerns and international migration. International Migration Review, p. 105-131, 1996.

KLEIN, Naomi. Esto lo cambia todo: El capitalismo contra el clima. Barcelona: Paidós, 2015.

MCLEMAN, Robert; SMIT, Barry. Migration as an adaptation to climate change. Climate Change, v. 76, p. 31-53, 2006.

MOUSLY MBAYE, Linguère. Climatic change, natural disasters, and migration. IZA World of Labor, n. 346, 2017.

ODRIOZOLA, Ignacio. Desplazados sin nombre: dificultades y desafíos en torno a una definición universal de "refugiados climáticos". Revista Jurídica de Buenos Aires, Fondo Editorial de Derecho y Economía, v. 42, n. 95, p. 87-112, 2017.

PENCHASZADEH, Ana Paula. Pervertibilidad de la condición de refugiado. RMF (Forced Migration Review), n. 36, p. 64-65, 2010.

WELZER, Harald. Guerras climáticas: Por qué mataremos (y nos matarán) en el siglo XXI. Madrid: Katz, 2010. 